\title{
Understanding the Biology and Pathogenesis of the Kidney by Single-Cell Transcriptomic Analysis
}

\author{
Yuting Ye Hui Song Jiong Zhang Shaolin Shi \\ National Clinical Research Center for Kidney Diseases, Jinling Hospital, Nanjing University School of \\ Medicine, Nanjing, China
}

\section{Keywords}

Single-cell RNA-seq · Gene expression dynamics · Kidney ·

Cell type identification - Cell subpopulation

\begin{abstract}
Background: Single-cell RNA-seq (scRNA-seq) has recently emerged as a revolutionary and powerful tool for biomedical research. However, there have been relatively few studies using scRNA-seq in the field of kidney study. Summary: scRNA-seq achieves gene expression profiling at single-cell resolution in contrast with the conventional methods of gene expression profiling, which are based on cell population and give averaged values of gene expression of the cells. Single-cell transcriptomic analysis is crucial because individual cells of the same type are highly heterogeneous in gene expression, which reflects the existence of subpopulations, different cellular states, or molecular dynamics, of the cells, and should be resolved for further insights. In addition, gene expression analysis of tissues or organs that usually comprise multiple cell types or subtypes results in data that are not fully applicable to any given cell type.
\end{abstract}

scRNA-seq is capable of identifying all cell types and subtypes in a tissue, including those that are new or present in small quantity. With these unique capabilities, scRNA-seq has been used to dissect molecular processes in cell differentiation and to trace cell lineages in development. It is also used to analyze the cells in a lesion of disease to identify the cell types and molecular dynamics implicated in the injury. With continuous technical improvement, scRNA-seq has become extremely high throughput and cost effective, making it accessible to all laboratories. In the present review article, we provide an overall review of scRNA-seq concerning its history, improvements, and applications. In addition, we describe the available studies in which scRNAseq was employed in the field of kidney research. Lastly, we discuss other potential uses of scRNA-seq for kidney research. Key Message: This review article provides general information on scRNA-seq and its various uses. Particularly, we summarize the studies in the field of kidney diseases in which scRNA-seq was used and discuss potential additional uses of scRNA-seq for kidney research.

(c) 2018 S. Karger AG, Basel

\section{KARGER}

(c) 2018 S. Karger AG, Basel 


\section{Introduction}

Gene expression profiling is a routine approach to dissect the molecular mechanism underlying physiological and pathological processes. People have to use tissues and even organs which consist of many cell types for gene expression studies due to the requirement of a large amount of RNA in microarray or RNA-seq analysis. This "bulk" gene expression profiling has obvious drawbacks in that the expression level of a gene is the averaged value of all individual cells of the same or different cell types and that the alterations of gene expression may occur in different cells but are considered to be in the same ones and interact with each other, resulting in misinterpretation of the data. Therefore, examining gene expression in single cells has long been desired by researchers, and efforts to achieve this have been made over the last decades [1].

The importance of single-cell gene expression analysis includes (1) more accurate interpretation of gene expression data in individual cells, particularly concerning the interactions of genes with altered expression, (2) identification of cell types, including new cell types or subtypes, that are involved in disease progression, and (3) acquisition of gene expression snapshots during cellular transition from one state to another, enabling identification of activated regulatory network and signaling pathways at a particular cellular state.

In this review article, we will describe (1) the history of single-cell analysis, (2) the development of single-cell RNA-seq (scRNA-seq) technology, (3) the major uses of scRNA-seq, (4) various scRNA-seq analyses coupled with other features and their uses, (5) current studies of the kidney using scRNA-seq, and (6) perspectives on scRNAseq for kidney research.

\section{Brief History of Single-Cell Gene Expression Analysis}

A typical cell has less than 1 pg of mRNA, making it extremely difficult to analyze its gene expression. To overcome sample insufficiency of mRNA from single cells, Eberwine et al. [1] designed an approach to amplify mRNA by microinjecting a primer tagged with T7 promoter sequence, nucleotides, and enzymes to a living neuronal cell such that mRNA can be converted to cDNA. The T7 promoter on each cDNA molecule then drives RNA synthesis, resulting in amplification of RNA over a million-fold. Unfortunately, since there was no highthroughput assay (e.g., microarray or RNA-seq) for global gene expression at that time, the amplified RNA had to

Understanding the Biology and

Pathogenesis of the Kidney be used for detection of the expression of genes of interest by probes or PCR. Several pieces of other earliest work of single-cell analysis were also performed to examine the expression of a limited number of genes of interest [2-4]. It should be pointed out that these earliest studies all noticed that morphologically identical cells were heterogeneous in gene expression.

A decade later, microarray technology was developed for gene expression analysis, making global single-cell gene expression profiling possible. Tietjen et al. [5] performed single-cell cDNA PCR amplification and analyzed the products with microarray. They found that gene expression during the differentiation from an olfactory progenitor cell to a mature sensory neuron is highly dynamic. A similar approach was taken to profile single-cell gene expression during pancreatic development, and numerous novel genes and several key signaling pathways involved in the development of the pancreas were identified [6]. Several years later, scRNA-seq was developed.

\section{The Development of scRNA-seq Technology}

With the advent of RNA-seq technology [7], scRNAseq has replaced single-cell microarray for the obvious advantages of RNA-seq over DNA microarray, including resolution at a single nucleotide, ability to distinguish different isoforms and allelic expression, possibility to identify new transcripts, low sample quantity requirement, and low cost [8]. Tang et al. [9, 10] established the scRNAseq method and applied it to single blastocyst cells analysis. With scRNA-seq, they detected more expressed genes compared with microarray and identified novel genes; in addition to blastocysts, they also performed scRNA-seq on oocytes lacking Dicer.

In scRNA-seq, RNA needs to be converted to cDNA, which is then amplified for sufficient quantity for sequencing. For amplification, the Smart-seq method was designed in which full-length mRNA is reverse transcribed followed by cDNA amplification using PCR. This method allows identification of alternative transcript isoforms and single nucleotide polymorphisms [11, 12]. Alternatively, a method termed CEL-seq has been developed in which barcode- and T7 promoter-containing primers are used to reverse transcribe each mRNA molecule into cDNA, followed by in vitro transcription of complement RNA, resulting in a sufficient amount of cRNA for sequencing [13].

Although scRNA-seq can be used for single-cell gene expression profiling, in the early studies of scRNA-seq 
each single-cell sample had to be handled individually, including single cell collection, cell lysis, RNA extraction, and cDNA synthesis and amplification. This made simultaneous scRNA-seq of a large number of cells difficult and even impossible. The manually operating procedure typically allowed only several dozen single cells being processed at a time. High-throughput methods for single cell isolation and processing on a large scale were highly desired.

In 2013, the Fluidigm C1 Auto Single-cell Separation and cDNA Prep system was launched. This is a microfluidic system that traps single cells in 96 or 384 chambers each run, followed by cell lysis, RNA reverse transcription, and cDNA PCR amplification $[14,15]$. The cDNA generated from each single cell is sufficient for library construction and sequencing. Although this is the first automatic system for scRNA-seq, much more efficient systems have soon developed, e.g., Drop-seq [16, 17].

Drop-seq is a platform in which individual cells are separated into nanoliter-sized aqueous droplets and then mixed with a unique barcode to label the cDNA molecules from each cell identically by the unique barcode. Then the cDNA from thousands of cells can be mixed, amplified, and sequenced simultaneously. The resulting sequences are sorted according to their unique barcode for each single cell. This platform is high throughput with extremely low cost $[16,17]$.

Most recently, several variant methods have been created, e.g., microwell-seq $[18,19]$, in which a single-cell suspension is added onto a gel plate containing thousands of wells on the surface with a size for just one cell. After single cells have been trapped in the individual microwells, magnetic beads bound with oligos that contain the sequences of barcode, unique molecule index, and poly $(\mathrm{dT})$ are added onto the gel. Due to the limited space of the microwells with one cell, only one bead is allowed to get into the well to capture the mRNA molecules of the cell through poly(A). Next, the beads are collected and combined, and subjected to cDNA reverse transcription and amplification followed by sequencing.

Most recently, Rosenberg et al. [20] created a simpler, but more powerful, method for scRNA-seq which they call SPLit-seq. This method is suitable for fixed cells and nuclei and uses the cells or nuclei themselves as the reaction chamber (not like other systems that use chambers, microwells, or aqueous/oil droplets) for cDNA preparation. All cells are then pooled for CDNA PCR amplification and sequencing. The idea for this method to distinguish RNA/cDNA molecules from different cells is that the cells or nuclei are randomly divided into wells and mixed with RT reagents with well-specific barcoded RT primers for cDNA synthesis, and then the cells in different wells are pooled and split into wells for second barcoding. Repeat the splitting and barcoding for another two cycles. The total of four times of splitting and barcoding is sufficient to make the cDNA molecules in one cell labeled differently from those in another cell. Finally, the cells are pooled for cDNA extraction, amplification, and sequencing. The resulting sequences are sorted based on cell-specific multiplexed barcodes. This method does not require custom equipment (e.g., Fluidigm $\mathrm{C} 1$ and 10X Genomics, etc.), making it accessible to all ordinary molecular biology laboratories at a low cost.

\section{Major Uses of scRNA-seq}

\section{Identification of Cell Types in Tissues/Organs and} Subpopulations of the Same Cell Type

One of the major uses of scRNA-seq is to dissect the cell types in tissues or organs. This kind of work usually results in identification of new cell types according to their differential and characteristic gene expression. scRNA-seq is extremely useful and probably the only way for identification of new cell types that are rare in quantity in a complex tissue. scRNA-seq analysis is also capable of identifying subpopulations of a known cell type, which are indistinguishable in morphology but do possess distinct gene expression signatures and functions. With this capability, scRNA-seq has brought a revolution to biomedical research. In recent years, a large number of new cell types and subpopulations in various tissues or organs has been identified in an amazingly quick manner. Understanding the roles of new cell types or subpopulations would certainly provide new insights into the physiological and pathological processes and is the direction and focus of future studies.

Shalek et al. [21] performed scRNA-seq on mouse bone-marrow-derived dendritic cells and found that they were heterogeneous in gene expression with a correlation of 0.29-0.62. They also found that when these cells were treated with lipopolysaccharide, they exhibited extensive bimodal variation in mRNA abundance of genes (including hundreds of key immune genes) and splicing patterns among the cells, suggesting the presence of distinct states or a subpopulation of the bone-marrow-derived dendritic cells [21]. Besides this work, Jaitin et al. [22] used scRNAseq to analyze splenic tissues and achieved marker-free decomposition of the tissue into multiple immune cell types, including distinct subpopulations of dendritic cells. 
scRNA-seq has also been used to analyze several other tissues or organs, including sensory neurons [23], forebrain neurogenic zone [24], hematopoietic stem cells [25], intestine [26], cortical region [27], retinal bipolar neurons [28], and blood cells [29], resulting in identification of a large number of new cell types or subpopulations from these tissues.

\section{Cell Differentiation and Cell Lineage Analysis in Development}

Since scRNA-seq can identify any differences in gene expression among individual cells even of the same type, it is apparently the best way to track cellular state transitions during differentiation and to map cell lineages in development.

An open and fundamental question in embryonic development is when the blastomeres start to become different concerning gene expression and cell fate. It was generally thought that the earliest time for blastomere differentiation is the 8- or 16-cell stage. However, there was lack of evidence, particularly molecular evidence, for this speculation. Recently, Biase et al. [30] profiled single blastomeres in mouse 2- and 4-cell embryos and found distinct gene expression between the blastomeres even in 2 -cell embryos. They further found that the distinct gene expression signature of the two blastomeres in 2-cell embryos can be passed down to the cells in the inner cell mass and trophectoderm, suggesting that the earliest cell differentiation in embryonic development takes place at the 2-cell stage. Huang et al. [31] performed a similar study, but concluded that the earliest differentiation of blastomeres is at the 4-cell stage.

Treutlein et al. [32] performed scRNA-seq analysis of the lung at different developmental stages. Based on the results, they classified lung cells into distinct groups, not only having confirmed the classical epithelial cell types in the distal lung, but having also discovered many other cell types or subpopulations. They further reconstructed lineage hierarchies of distal lung epithelium according to these findings.

By scRNA-seq, Pollen et al. [33] identified multiple new subpopulations of cells in the developing cortex. Later, Wang et al. [34] used scRNA-seq to analyze human developing neurons and identified distinct cell subpopulations and the determining regulators that govern their bifurcation.

scRNA-seq lineage analyses in development have been conducted for many developmental processes, e.g., myeloid progenitor cell development $[35,36]$, heart development [37-39], hematopoiesis [40-42], early mesoderm
[43], and mouse early gastrulation [44]. In these studies, in addition to identification of new cell types or subtypes in the developmental processes, continuous gene expression spectrums were also snapshot at different stages of cell differentiation [45-48].

Most recently, Raj et al. [49] designed a more precise cell differentiation and lineage tracing approach that is based on CRISPR-Cas9 and scRNA-seq. They applied it to the study of zebrafish brain development and established lineage trees of brain development. This system uses transgenic multiple barcodes and inducible Cas9 expression such that Cas9 can be inducibly activated at multiple time points in development. As a result, the multiple barcodes were partially edited to mark the cells at different time points of cell differentiation and brain development, such that the relationship of brain cells in development can be clearly shown by the pattern of edited barcodes. They call this method scGESTALT.

\section{Diseases}

Like its uses in identifying cell types or subpopulations, tracing cell lineages in development, and snapshoting gene expression of cells in transition, scRNA-seq can also be used to decompose cell types, including the rare ones, in the lesions of disease, or to determine the cell trajectory in injury through the changes in gene expression, signaling pathways, and interaction network in the cells.

Patel et al. [50] performed scRNA-seq on primary glioblastoma and found that the cells were different in expression of the genes that are involved in oncogenesis, proliferation, immune response, hypoxia, and stemness; based on this, they were able to classify subtypes of the cells of glioblastoma. Similarly, metastatic melanoma was dissected by scRNA-seq and heterogeneity was observed among the cells, with some expressing genes of drug resistance program while some others did not. In addition, the presence of other cell types, including immune, stromal, and endothelial cells, was also identified, thus having provided novel insights into the multicellular ecosystem of melanoma [51]. Recently, scRNA-seq analysis was conducted on stem cells in chronic myeloid leukemia and revealed subpopulations of leukemia cells that are resistant to drug treatment and have distinct molecular signatures in gene expression [52].

Circulating tumor cells (CTCs) are very useful for diagnosis and efficacy evaluation in the treatment of cancers. Miyamoto et al. [53] analyzed CTCs from patients with prostate cancer and found that they were highly heterogeneous in gene expression and that some CTCs were special in that Wnt signaling was activated in them. Sur- 
prisingly, further experiments showed that the activation of Wnt signaling in this fraction of CTCs underlay their resistance to drug treatment.

scRNA-seq of Th17 cells isolated from the central nervous system and lymph nodes at the peak of autoimmune encephalomyelitis has revealed the genes underlying pathogenicity and disease susceptibility [54].

\section{Genetics}

scRNA-seq can be used for genetic studies. One example is the identification of cell type-specific cis-eQTLs and coexpression QTLs in individual cells, which can provide a more precise relationship between cis-eQTL and coexpression QTLs. Van der Wijst et al. [55] conducted scRNA-seq on 25,000 peripheral blood mononuclear cells from 45 donors and identified not only the previously reported cis-eQTLs, but also new cell typespecific cis-eQTLs. They further generated personalized coexpression networks and identified genetic variants that significantly alter coexpression relationships. They termed this approach single-cell eQTL analysis.

Simultaneous transcriptomic, genomic, and epigenetic (DNA methylation) analyses of single cells can determine more precisely the "cause and effect" relationship between the genetic makeup and gene expression (even phenotypes, e.g., cancer). We will exemplify and discuss this issue in more detail later.

\section{scRNA-seq Coupled with Various Other Features}

A variety of methods and research approaches that are based on scRNA-seq have been developed for different purposes, leading to greatly extended use of scRNA-seq. Although these methods or approaches have not been tested with the kidney, they will certainly find their use in kidney research. In the following section, we will briefly describe these variant scRNA-seq methods.

\section{Simultaneous Transcriptomic, Genomic, and}

\section{Epigenomic Analyses of Single Cells}

Several studies have developed methods that enable simultaneous genomic, epigenomic, and transcriptomic analyses in single cells. These methods include the one, termed scTrio-seq, which can acquire data of genomic copy number variations, DNA methylome, and transcriptome from single cells [56]. scTrio-seq analysis of cancer cells identified new subpopulations in hepatocellular carcinoma [56]. Another study that analyzed single nucleotide polymorphism as well as genomic DNA meth- ylome and transcriptome in single cells (called scMT-seq) was also performed [57].

Clark et al. [58] developed scNMT-seq, which allows simultaneous analyses of chromatin accessibility as well as DNA methylation and transcription in single cells. To map the sites of chromatin accessibility, GpC methyltransferase is used to label the open chromatin, followed by bisulfite and RNA sequencing. They analyzed mouse differentiating embryonic stem cells and found the links between the three sets of data and dynamic coupling between epigenomic layers during differentiation.

\section{Simultaneous Transcript and Protein Analysis}

It is known that protein levels are frequently inconsistent with the corresponding mRNA levels due to complex regulatory mechanisms that may interfere with each other. Although scRNA-seq is powerful in retrieving transcriptomic information from single cells, the heterogeneity information of proteins, which is associated with phenotypes more closely than RNA in individual single cells, is also desirable. Unfortunately, global protein profiling for single cells is currently not feasible. Nevertheless, efforts have been made to quantify a few proteins of interest during scRNA-seq. Stoeckius et al. [59] designed CITEseq (cellular indexing of transcriptomes and epitopes by sequencing). In this method, antibodies against multiple cell surface epitopes are labeled with oligonucleotides and then used to bind cells to be subject to scRNA-seq. The amount of antibody that is bound to the cell surface (which can be determined by the amount of oligonucleotides) reflects the amount of the corresponding proteins on the cell surface of single cells. These cells were subject to scRNA-seq, and the oligonucleotides linked to the antibodies were also sequenced, resulting in quantification of the proteins on the surface of individual single cells. Peterson et al. [60] conducted a similar study (REAP-seq) in which cells to be subject to scRNA-seq are first bound with 82 oligonucleotide barcoded antibodies such that the proteins to be detected can be quantified by the amount of specific oligonucleotides. In the study, they identified new cell types of CD8+ lymphocytes that have differential responses to CD27 agonist costimulation.

\section{Noncoding RNA Sequencing and Profiling in Single Cells}

Typical methods for scRNA-seq require cDNA synthesis which is primed by oligo-dT that anneals to the poly(A) tail of mRNA molecules. However, for many noncoding RNAs which do not have a poly (A) tail at the $3^{\prime}$ end, they are ignored by current methods of scRNA- 
seq. To solve this problem, Fan et al. [61] designed a method, called SUPeR-seq, for universal poly(A)-independent RNA sequencing. In this method, both polyadenylated and non-polyadenylated RNAs in individual single cells can be measured. Another method, called random displacement amplification sequencing (RamDAseq), has been also developed for non-polyadenylated RNA, including enhancer RNA sequencing [62]. Moreover, Faridani et al. [63] designed a method that is specific for small RNA, including miRNA, tRNA, and small nucleolar RNA.

\section{Using scRNA-seq to Capture Rare Cell Populations or} Molecular Dynamics

scRNA-seq can identify cell types in tissues or capture molecular states in a cellular process. However, when the particular cell types are rare in quantity or the cell molecular states are very short temporally, it will be difficult for scRNA-seq to capture them. Habib et al. [64] developed a technique, which is called Div-seq, to enrich and identify a small amount of dividing neurons. They first pulse labeled proliferating neuronal cells by 5-ethynyl-2'-deoxyuridine (EdU) followed by nuclei isolation and fluorescence-activated cell sorting by using EdU fluorescence. The nuclei with fluorescence, which were from proliferating cells or the cells that were just divided, were subject to RNA-seq (i.e., single-nucleus RNA-seq [sNuc-seq]). This method can sensitively distinguish cells that are at different stages of proliferation, enabling detection of transcriptional dynamics in proliferating cells.

\section{scRNA-seq Coupled with Functional Analysis Using CRISPR}

Another important use of scRNA-seq is to dissect molecular circuits in cells by inactivating transcription factors using CRISPR technology followed by scRNA-seq. Dixit et al. [65] infected cells with viruses expressing sgRNAs that target a couple of tens of transcription factors. The virus titer was optimized such that only one or two transcription factors could be inactivated in each single cell. They then performed scRNA-seq on these cells and analyzed with bioinformatics. Eventually they generated molecular circuits based on the differential alterations of gene expression in response to lipopolysaccharide in the cells that had different transcription factors deleted. Similar studies were conducted to dissect the regulatory circuits in endoplasmic reticulum homeostasis, immune cells, and other cell types by using CRSPRi followed by scRNA-seq [66-68].

Understanding the Biology and Pathogenesis of the Kidney

\section{Current Studies in the Field of Kidney Research That Used scRNA-seq}

Single renal cell analysis was attempted two decades ago. However, due to technical limitations, the studies were based on RT-PCR analysis of the expression of known genes. Schröppel et al. [69] established a method for single-podocyte gene expression analysis, including collection of mouse glomerular podocytes and optimization of RT-PCR. This method was shown to work well in detecting genes known to be specifically expressed in podocytes. Huber et al. [70] isolated the cells at the ureteric bud tip of mouse embryos on embryonic day 17 (E17) and analyzed transcripts of $\mathrm{Cl}$ channel 2. They found that only a portion of the examined cells expressed this gene. Nissant et al. [71] performed RT-PCR analysis of the expression of chloride channels in distal convoluted tubules and found that their expressions are heterogeneous in the cell type. Except for these early studies of single renal cell analysis, there was essentially no further work employing single-cell analysis to study renal physiololgy and pathogenesis until recently when the highthroughput scRNA-seq technology became available.

Lu et al. [72, 73] used the Fluidigm C1 platform for single glomerular cell capture, lysis, RNA extraction, as well as cDNA synthesis and amplification in the microfluidic system. They determined the identity of each cell by PCR detection of the markers for podocytes or mesangial cells and then sequenced the cDNA. They found that both mesangial cells and podocytes are extremely heterogeneous in gene expression, with correlation coefficients of 0.20 (mesangial cells) and 0.25 (podocytes), respectively $[72,73]$. They next identified genes expressed in every single mesangial cell or podocyte examined, and speculated that they are either essential for mesangial cells or podocytes to survive (therefore, being house-keeping genes) or essential for the unique structure and function of the cell types, without which the organism will not survive. Particularly, in mesangial cells they found high enrichment of genes involved in endothelial activity, supporting the long-existing notion that mesangial cells are specialized pericytes. Interestingly, they found that some mesangial cells express the marker genes of podocytes (e.g., Wt1) and of endothelial cells (Tie2, Flk1, Flt1/ Vegfr1) [72]. In podocyte scRNA-seq, by removing house-keeping genes that are also expressed in other glomerular cell types, they identified 92 genes that are specifically expressed in all individual podocytes. They speculated that these genes are candidates of podocyte-specific essential genes. They next tested the importance of 37 
genes for podocyte cytoskeletons in cultured podocytes. The cytoskeleton was chosen for testing because podocytes have special cytoskeletons to make their unique structure and fulfill their fundamental role as part of the glomerular filtration barrier, and therefore the genes specifically essential for podocytes are highly likely associated with cytoskeletons. They found that deficiency of 30 of the 37 genes resulted in cytoskeletal injury of podocytes, suggesting that most of the 92 candidate genes are truly podocyte-specific essential genes and mainly involved in the maintenance of cytoskeletons for podocyte unique structure. This finding is consistent with the fundamental role of podocytes in the glomerular filtration barrier [73]. Their work based on scRNA-seq has greatly facilitated the identification of genes specifically essential for mesangial cells and podocytes.

Der et al. [74] performed scRNA-seq to dissect the molecular heterogeneity in lupus nephritis. scRNA-seq successfully distinguished not only the cell types of proximal tubules, loop of Henle, distal tubules, and collecting ducts, but also found that interferon scores based on tubular epithelial cells' scRNA-seq data of lupus nephritis patients correlate with renal clinical scores. Tang et al. [75] performed scRNA-seq analysis of zebrafish kidney that was deficient in several genes and investigated the consequence of the gene deficiencies; in addition, they uncovered several novel cell types.

Chen et al. [76] performed scRNA-seq on mouse renal collecting duct cells and created the database from which genes selectively expressed in each cell type of collecting duct can be found. Furthermore, they showed that a small fraction of hybrid cells expressed aquaporin-2 and anion exchanger 1 or pendrin transcripts. They additionally showed that mRNAs of receptors and their ligands were localized in different cells; for example, the receptor Notch2 was found in principal cells, while the ligand Jag1 was present in intercalated cells. These observations suggest a signaling cross-talk between these cell types.

Adam et al. [77] performed scRNA-seq on developing mouse kidney and generated a gene expression atlas of newborn mouse kidney at single-cell resolution. Magella et al. [78] also performed scRNA-seq of developing mouse kidney and unexpectedly identified the expression of Gdnf, a key driver of branching morphogenesis of the collecting duct system, in nephrogenic zone stromal cells. GDNF was previously thought to be solely from the cap mesenchyme nephron progenitors.

Recently, Park et al. [79] performed scRNA-seq analysis of 57,979 cells from healthy mouse kidney and identified 18 previously defined cell types and three new cell types. In addition, based on the scRNA-seq data, they inferred that inherited kidney diseases that arise from distinct genetic mutations but share the same phenotypic manifestation originate from the same cell type. They also noticed that collecting duct generates a spectrum of cell types via a new transitional cell type. Furthermore, by cell trajectory and lineage tracing analyses, they found that Notch signaling mediates the transitions of intercalated cells and principal cells; in disease the transitions were shifted towards a principal cell fate.

Mouse glomeruli have just been analyzed by scRNAseq at a large scale with a total of 13,000 glomerular cells being sequenced, resulting in mouse glomerular singlecell transcriptome atlas [80]. In this work, the authors identified three subpopulations of podocytes as well as a subset of endothelial cells exhibiting proliferative property as shown by expression of proliferation marker genes.

\section{Perspectives}

scRNA-seq has emerged as an extremely powerful tool for dissecting molecular processes in differentiation, structural and functional homeostasis, and injury of cells. In addition, it precisely and efficiently decompose the complex tissues concerning cell type makeup, leading to much better understanding of physiological and pathological mechanisms. Due to its power, scRNA-seq has rapidly become a routine tool for researchers, and the publications based on scRNA-seq technology have increased exponentially in the past several years. In the field of kidney research, however, the use of scRNA-seq appears not as adequate as in other fields (e.g., neuroscience, immunology, development, and cancer), as suggested by only a few publications of kidney research in which scRNA-seq was used. This limited number of studies has only exemplified the power of scRNA-seq for kidney research. There are many fundamental issues in kidney biology and diseases that can be addressed by and even only by scRNA-seq.

First of all, we still have not obtained the full atlas of single cell gene expression of all renal cell types of both normal mouse and human. Park et al. [79] performed the largest scale of kidney cell scRNA-seq so far with $>50,000$ cells sequenced, and they identified 18 previously known renal intrinsic and immune cell types. In addition, they distinguished subpopulations of several known cell types and identified three new cell types in kidney. Nevertheless, further efforts are required to confirm additional previously known cell types and determine their molecu- 
lar definition. Moreover, scRNA-seq will certainly identify more new cell types, especially subtypes, because it is known that there are more than 50 different compartments, segments, cell masses, etc. in a kidney (https:// en.wikipedia.org/wiki/Glomerulus_(kidney)\#Sources), therefore the total number of cell types in kidney will be surely not smaller than 50 . The aforementioned study by Park et al. [79] missed many other known cell types and thus likely additional new cell types or cell subtypes. The reason for their failure to detect the full spectrum of cell types could be due to the fact that many new cell types or subtypes have a very small proportion in entire cell mass of a kidney, making the 50,000 scRNA-seq not sufficient for detecting the rare cell types or subtypes. Another reason could be that many cell type may be vulnerable to the procedure for kidney cell dissociation and separation, leading to death and RNA degradation that might account for the failure of about $25 \%$ of single kidney cells in sequencing in the work by Park et al. [79] (only 43,745 out of 57,979 cells passed quality control and were analyzed). To overcome these potential problems, it is feasible to dissect only a specific portion of kidney tissues (e.g., proximal tubules or glomeruli) and to dissociate cells with an improved method for increased cell viability, such that the complexity of cell types will be reduced and cell viability will be increased, allowing the rare cell types to be picked up and analyzed. Indeed, Chen et al. [76] focused on only collecting duct for scRNA-seq and resolved the A-type intercalated cells, the B-type intercalated cells, principal cells, and hybrid cells that are between A- and B-type intercalated cells in gene expression. This approach gave rise to more precise dissection of cell types or subtypes compared with scRNA-seq of whole kidney, as described in the work by Park et al. [79]. Another example is the scRNA-seq of mouse glomeruli by Karaiskos et al. [80]. They resolved three subpopulations of podocytes in contrast with Park et al.'s [79] work based on scRNA-seq of entire kidney. In conclusion, a lot of work is required to dissect the cell types or subtypes in a kidney, particularly for the rare cell types or subtypes at a higher resolution.

Another important direction is the resolution of cell lineages during kidney development. The kidneys of E14.5 and newborn mouse embryos were analyzed by scRNA-seq [77, 78], and 9 and 16 distinct cell types, respectively, were identified in those studies. However, the cell types and lineages at other developing stages are still lacking and deserve to be further explored to obtain more detailed views of molecular and cell lineage transitions during kidney development.

Understanding the Biology and

Pathogenesis of the Kidney
It has been mentioned earlier that scRNA-seq can be used to dissect pathological processes. Renal biopsies from lupus nephritis patients were analyzed by scRNAseq, and it was found that interferon scores from tubular epithelial cells' scRNA-seq data from lupus nephritis patients correlate with renal clinical scores [74]. Essentially, scRNA-seq can be used for any kind of kidney disease. By scRNA-seq analysis of the kidney at different stages of disease, the cell types or subtypes involved in the process, as well as the cellular states and molecular dynamics towards injury, would be revealed. In fact, scRNA-seq should be the only way to acquire this information. We expect that the available gene expression profiles obtained through conventional "bulk" microarray or RNA-seq will be replaced by a single-cell gene expression atlas based on scRNA-seq in the near future due to the intrinsic shortcomings of "bulk" profiling that include its inability of resolving gene expression in cell types or subtypes, capturing cellular states and molecular dynamics of a cell type or subtype towards differentiation or injury, and mapping molecular events to individual cells, as well as its requirement of a large number of cells for analysis (which is particularly problematic for human biopsies).

Elucidation of the origins of new cells in renal cell regeneration after injury or the extra cells that are part of a lesion (e.g., the crescents in glomerulonephritis) is certainly important with respect to clinical significance. There have been studies showing that tubular epithelial cells can be regenerated from certain intrarenal cells to compensate the cell loss in renal injury [81], that tubular epithelial cells can switch to fibroblast cells to contribute to renal fibrosis [82], and that some parietal epithelial cells have stem cell activity and can differentiate into podocytes upon glomerular injury and podocyte loss [83]. These observations were made mainly by transgenically labeling certain cell types and tracing their fates. This approach requires the use of known cell type-specific markers. However, there are several caveats in such studies in that many cell type-specific markers are not specific enough, as shown in the recent work by Lu et al. [72]; besides, cell fate tracing in vivo requires a cell typespecific promoter and, unfortunately, the specificity of such promoters is of concern (e.g., activity leaking), as detailed in a review article by Romagnani et al. [84]. In this scenario, the use of scRNA-seq would be critical and even the only solution for such kind of studies. scRNAseq analysis is capable of identifying the cell types, including those that are rare, in the renal lesions, and it is also capable of determining the trajectory of cell transition 
with respect to molecular dynamics, thereby precisely mapping the origins of newly generated cells.

Although the essential genes of mouse mesangial cells and podocytes, including the cell type-specific essential genes that govern the unique structure and function of mesangial cells and podocytes, have been identified by scRNA-seq with a specially designed approach $[72,73]$, similar work will have to be conducted for all renal cell types or subtypes in order to better understand the biology and pathogenesis of the cell types in kidney.

Lastly, regardless of the rapid improvement of the scRNA-seq technology, there are still some technical limitations and challenges that researchers need to be aware of in the use of scRNA-seq. (1) Due to the small amount of mRNA in a cell, the transcripts of only hundreds to thousands of genes are typically detected in most studies. This issue could be partly addressed by increasing the depth of sequencing. (2) The preparation of single-cell suspension can be difficult and lengthy, depending on the tissue types or organs, resulting in alteration of gene expression during the preparation of single-cell suspension. Thus, efforts must be made to optimize the procedure for quick single cell preparation. (3) Due to mRNA sequence feature and structure, the reverse transcription efficiency of different mRNA molecules is differential, resulting in biased representation of cDNA in scRNA-seq; in addition, mRNAs in each single cell are so little that their cDNAs need to be amplified by PCR, a technique that always gives rise to amplification bias depending on sequence feature and abundance of the cDNA. These two steps of RT-PCR both affect the accuracy of gene expression profiling by scRNA-seq. (4) scRNA-seq involves reverse transcription of mRNA, and oligo-dT is usually used to prime cDNA synthesis on polyadenylated mRNA. However, there are many mRNA species that do not possess a poly(A) tail at the $3^{\prime}$ end, making their reverse transcription into cDNA impossible, and thus their presence is ignored in scRNA-seq. (5) Unlike "bulk" gene profiling using cell population, it is very difficult to investigate the genome-wide correlation of mRNA levels with that of corresponding proteins in the same cells. (6) Due to the limited amount of materials in a cell for analysis, it is difficult to perform multiple omics analyses with the same cell in contrast with cell populations for which multiple omics analyses are feasible and easy. (7) Since scRNA-seq requires a relatively large number of cells for the automatic system of cell separation and capture, it will be challenging when human biopsies from patients are to be used for scRNA-seq. Nevertheless, attempts have been made to solve these problems, e.g., some studies have performed multiple omics analysis in single cells [56-60], and several studies have used specially designed methods to allow non-polyadenylated RNA species to be detected in scRNA-seq [61-63].

\section{Acknowledgments}

This work was supported by the National Natural Science Foundation of China $(81670653,81400722)$ and the National Key Research and Development Program of China (2016YFC0904103).

\section{Disclosure Statement}

The authors have no conflicts of interest to declare.

\section{References}

1 Eberwine J, Yeh H, Miyashiro K, Cao Y, Nair S, Finnell R, Zettel M, Coleman P: Analysis of gene expression in single live neurons. Proc Natl Acad Sci USA 1992;89:3010-3014.

2 Lambolez B, Audinat E, Bochet P, Crépel F, Rossier J: AMPA receptor subunits expressed by single Purkinje cells. Neuron 1992;9:247258.

3 Trümper LH, Brady G, Bagg A, Gray D, Loke SL, Griesser H, Wagman R, Braziel R, Gascoyne RD, Vicini S, et al: Single-cell analysis of Hodgkin and Reed-Sternberg cells: molecular heterogeneity of gene expression and p53 mutations. Blood 1993;81:3097-3115.
4 Brady G, Billia F, Knox J, Hoang T, Kirsch IR, Voura EB, Hawley RG, Cumming R, Buchwald M, Siminovitch K: Analysis of gene expression in a complex differentiation hierarchy by global amplification of cDNA from single cells. Curr Biol 1995;5:909-922.

5 Tietjen I, Rihel JM, Cao Y, Koentges G, Zakhary L, Dulac C: Single-cell transcriptional analysis of neuronal progenitors. Neuron 2003;38:161-175.

6 Chiang MK, Melton DA: Single-cell transcript analysis of pancreas development. Dev Cell 2003;4:383-393.
7 Nagalakshmi U, Wang Z, Waern K, Shou C, Raha D, Gerstein M, Snyder M: The transcriptional landscape of the yeast genome defined by RNA sequencing. Science 2008;320: 1344-1349.

8 Wang Z, Gerstein M, Snyder M: RNA-Seq: a revolutionary tool for transcriptomics. Nat Rev Genet 2009;10:57-63.

9 Tang F, Barbacioru C, Wang Y, Nordman E, Lee $\mathrm{C}, \mathrm{Xu} \mathrm{N}$, Wang $\mathrm{X}$, Bodeau J, Tuch BB, Siddiqui A, Lao K, Surani MA: mRNA-Seq whole-transcriptome analysis of a single cell. Nat Methods 2009;6:377-382. 
10 Tang F, Barbacioru C, Nordman E, Li B, Xu N, Bashkirov VI, Lao K, Surani MA: RNA-Seq analysis to capture the transcriptome landscape of a single cell. Nat Protoc 2010;5:516535.

11 Ramsköld D, Luo S, Wang YC, Li R, Deng Q, Faridani OR, Daniels GA, Khrebtukova I, Loring JF, Laurent LC, Schroth GP, Sandberg R: Full-length mRNA-Seq from singlecell levels of RNA and individual circulating tumor cells. Nat Biotechnol 2012;30:777782.

12 Picelli S, Faridani OR, Björklund AK, Winberg G, Sagasser S, Sandberg R: Full-length RNA-seq from single cells using Smart-seq2. Nat Protoc 2014;9:171-181.

13 Hashimshony T, Wagner F, Sher N, Yanai I: CEL-Seq: single-cell RNA-Seq by multiplexed linear amplification. Cell Rep 2012;2:666673.

14 Streets AM, Zhang X, Cao C, Pang Y, Wu X, Xiong L, Yang L, Fu Y, Zhao L, Tang F, Huang Y: Microfluidic single-cell whole-transcriptome sequencing. Proc Natl Acad Sci USA 2014;111:7048-7053.

15 Shalek AK, Satija R, Shuga J, Trombetta JJ, Gennert D, Lu D, Chen P, Gertner RS, Gaublomme JT, Yosef N, Schwartz S, Fowler B, Weaver S, Wang J, Wang X, Ding R, Raychowdhury R, Friedman N, Hacohen N, Park H, May AP, Regev A: Single-cell RNA-seq reveals dynamic paracrine control of cellular variation. Nature 2014;510:363-369.

16 Macosko EZ, Basu A, Satija R, Nemesh J, Shekhar K, Goldman M, Tirosh I, Bialas AR, Kamitaki N, Martersteck EM, Trombetta JJ, Weitz DA, Sanes JR, Shalek AK, Regev A, McCarroll SA: Highly parallel genome-wide expression profiling of individual cells using nanoliter droplets. Cell 2015;161:12021214.

17 Klein AM, Mazutis L, Akartuna I, Tallapragada N, Veres A, Li V, Peshkin L, Weitz DA, Kirschner MW: Droplet barcoding for singlecell transcriptomics applied to embryonic stem cells. Cell 2015;161:1187-1201.

18 Gierahn TM, Wadsworth MH, Hughes TK, Bryson BD, Butler A, Satija R, Fortune S, Love JC, Shalek AK: Seq-Well: portable, low-cost RNA sequencing of single cells at high throughput. Nat Methods 2017;14:395-398.

19 Han X, Wang R, Zhou Y, Fei L, Sun H, Lai S, Saadatpour A, Zhou Z, Chen H, Ye F, Huang $D, X u$ Y, Huang W, Jiang M, Jiang X, Mao J, Chen Y, Lu C, Xie J, Fang Q, Wang Y, Yue R, Li T, Huang H, Orkin SH, Yuan GC, Chen M, Guo G: Mapping the mouse cell atlas by Microwell-seq. Cell 2018;172:1091-1107.e17.

20 Rosenberg AB, Roco CM, Muscat RA, Kuchina A, Sample P, Yao Z, Graybuck LT, Peeler DJ, Mukherjee S, Chen W, Pun SH, Sellers DL, Tasic B, Seelig G: Single-cell profiling of the developing mouse brain and spinal cord with split-pool barcoding. Science 2018;360: 176-182.
21 Shalek AK, Satija R, Adiconis X, Gertner RS, Gaublomme JT, Raychowdhury R, Schwartz S, Yosef N, Malboeuf C, Lu D, Trombetta JJ, Gennert D, Gnirke A, Goren A, Hacohen N, Levin JZ, Park H, Regev A: Single-cell transcriptomics reveals bimodality in expression and splicing in immune cells. Nature 2013; 498:236-240.

22 Jaitin DA, Kenigsberg E, Keren-Shaul H, Elefant N, Paul F, Zaretsky I, Mildner A, Cohen N, Jung S, Tanay A, Amit I: Massively parallel single-cell RNA-seq for marker-free decomposition of tissues into cell types. Science 2014;343:776-779.

23 Usoskin D, Furlan A, Islam S, Abdo H, Lönnerberg P, Lou D, Hjerling-Leffler J, Haeggström J, Kharchenko O, Kharchenko PV, Linnarsson S, Ernfors P: Unbiased classification of sensory neuron types by large-scale singlecell RNA sequencing. Nat Neurosci 2015;18: 145-153.

24 Luo Y, Coskun V, Liang A, Yu J, Cheng L, Ge W, Shi Z, Zhang K, Li C, Cui Y, Lin H, Luo D, Wang J, Lin C, Dai Z, Zhu H, Zhang J, Liu J, Liu H, deVellis J, Horvath S, Sun YE, Li S: Single-cell transcriptome analyses reveal signals to activate dormant neural stem cells. Cell 2015;161:1175-1186.

25 Wilson NK, Kent DG, Buettner F, Shehata M, Macaulay IC, Calero-Nieto FJ, Sánchez Castillo M, Oedekoven CA, Diamanti E, Schulte $\mathrm{R}$, Ponting CP, Voet T, Caldas C, Stingl J, Green AR, Theis FJ, Göttgens B: Combined single-cell functional and gene expression analysis resolves heterogeneity within stem cell populations. Cell Stem Cell 2015;16:712724.

26 Grün D, Lyubimova A, Kester L, Wiebrands K, Basak O, Sasaki N, Clevers H, van Oudenaarden A: Single-cell messenger RNA sequencing reveals rare intestinal cell types. $\mathrm{Na}-$ ture 2015;525:251-255.

27 Tasic B, Menon V, Nguyen TN, Kim TK, Jarsky T, Yao Z, Levi B, Gray LT, Sorensen SA, Dolbeare T, Bertagnolli D, GoldyJ, Shapovalova N, Parry S, Lee C, Smith K, Bernard A, Madisen L, Sunkin SM, Hawrylycz M, Koch $\mathrm{C}$, Zeng H: Adult mouse cortical cell taxonomy revealed by single cell transcriptomics. Nat Neurosci 2016;19:335-346.

28 Shekhar K, Lapan SW, Whitney IE, Tran NM, Macosko EZ, Kowalczyk M, Adiconis X, Levin JZ, Nemesh J, Goldman M, McCarroll SA, Cepko CL, Regev A, Sanes JR: Comprehensive classification of retinal bipolar neurons by single-cell transcriptomics. Cell 2016; 166:1308-1323.e30.

29 Villani AC, Satija R, Reynolds G, Sarkizova S, Shekhar K, Fletcher J, Griesbeck M, Butler A, Zheng S, Lazo S, Jardine L, Dixon D, Stephenson E, Nilsson E, Grundberg I, McDonald D, Filby A, Li W, De Jager PL, Rozenblatt-Rosen O, Lane AA, Haniffa M, Regev A, Hacohen N: Single-cell RNA-seq reveals new types of human blood dendritic cells, monocytes, and progenitors. Science 2017;356:eaah4573.
30 Biase FH, Cao X, Zhong S: Cell fate inclination within 2-cell and 4-cell mouse embryos revealed by single-cell RNA sequencing. Genome Res 2014;24:1787-1796.

31 Huang W, Cao X, Biase FH, Yu P, Zhong S: Time-variant clustering model for understanding cell fate decisions. Proc Natl Acad Sci USA 2014;111:E4797-E4806.

32 Treutlein B, Brownfield DG, Wu AR, Neff NF, Mantalas GL, Espinoza FH, Desai TJ, Krasnow MA, Quake SR: Reconstructing lineage hierarchies of the distal lung epithelium using single-cell RNA-seq. Nature 2014;509: 371-375.

33 Pollen AA, Nowakowski TJ, Shuga J, Wang X, Leyrat AA, Lui JH, Li N, Szpankowski L, Fowler B, Chen P, Ramalingam N, Sun G, Thu M, Norris M, Lebofsky R, Toppani D, Kemp DW, Wong M, Clerkson B, Jones BN, Wu S, Knutsson L, Alvarado B, Wang J, Weaver LS, May AP, Jones RC, Unger MA, Kriegstein AR, West JA: Low-coverage single-cell mRNA sequencing reveals cellular heterogeneity and activated signaling pathways in developing cerebral cortex. Nat Biotechnol 2014;32: 1053-1058.

34 Wang J, Jenjaroenpun P, Bhinge A, Angarica VE, Del Sol A, Nookaew I, Kuznetsov VA, Stanton LW: Single-cell gene expression analysis reveals regulators of distinct cell subpopulations among developing human neurons. Genome Res 2017;27:1783-1794.

35 Paul F, Arkin Y, Giladi A, Jaitin DA, Kenigsberg E, Keren-Shaul H, Winter D, Lara-Astiaso D, Gury M, Weiner A, David E, Cohen N, Lauridsen FKB, Haas S, Schlitzer A, Mildner A, Ginhoux F, Jung S, Trumpp A, Porse BT, Tanay A, Amit I: Transcriptional heterogeneity and lineage commitment in myeloid progenitors. Cell 2016;164:325.

36 Drissen R, Buza-Vidas N, Woll P, Thongjuea S, Gambardella A, Giustacchini A, Mancini E Zriwil A, Lutteropp M, Grover A, Mead A, Sitnicka E, Jacobsen SEW, Nerlov C: Distinct myeloid progenitor-differentiation pathways identified through single-cell RNA sequencing. Nat Immunol 2016;17:666-676.

37 DeLaughter DM, Bick AG, Wakimoto $\mathrm{H}$ McKean D, Gorham JM, Kathiriya IS, Hinson JT, Homsy J, Gray J, Pu W, Bruneau BG, Seidman JG, Seidman CE: Single-cell resolution of temporal gene expression during heart development. Dev Cell 2016;39:480-490.

38 Liu Z, Wang L, Welch JD, Ma H, Zhou Y, Vaseghi HR, Yu S, Wall JB, Alimohamadi S, Zheng M, Yin C, Shen W, Prins JF, Liu J, Qian L: Single-cell transcriptomics reconstructs fate conversion from fibroblast to cardiomyocyte. Nature 2017;551:100-104.

39 Lescroart F, Wang X, Lin X, Swedlund B, Gargouri S, Sànchez-Dànes A, Moignard V, Dubois C, Paulissen C, Kinston S, Göttgens B, Blanpain C: Defining the earliest step of cardiovascular lineage segregation by single-cell RNA-seq. Science 2018;359:1177-1181.
Understanding the Biology and

Pathogenesis of the Kidney
Kidney Dis 2018;4:214-225

DOI: $10.1159 / 000492470$ 
40 Macaulay IC, Svensson V, Labalette C, Ferreira L, Hamey F, Voet T, Teichmann SA, Cvejic A: Single-cell RNA-sequencing reveals a continuous spectrum of differentiation in hematopoietic cells. Cell Rep 2016;14:966977.

41 Zhou F, Li X, Wang W, Zhu P, Zhou J, He W, Ding M, Xiong F, Zheng X, Li Z, Ni Y, Mu X, Wen L, Cheng T, Lan Y, Yuan W, Tang F, Liu B: Tracing haematopoietic stem cell formation at single-cell resolution. Nature 2016; 533:487-492.

42 Rodriguez-Fraticelli AE, Wolock SL, Weinreb CS, Panero R, Patel SH, Jankovic M, Sun J, Calogero RA, Klein AM, Camargo FD: Clonal analysis of lineage fate in native haematopoiesis. Nature 2018;553:212-216.

43 Scialdone A, Tanaka Y, Jawaid W, Moignard V, Wilson NK, Macaulay IC, Marioni JC, Göttgens B: Resolving early mesoderm diversification through single-cell expression profiling. Nature 2016;535:289-293.

44 Mohammed H, Hernando-Herraez I, Savino A, Scialdone A, Macaulay I, Mulas C, Chandra T, Voet T, Dean W, Nichols J, Marioni JC, Reik W: Single-cell landscape of transcriptional heterogeneity and cell fate decisions during mouse early gastrulation. Cell Rep 2017;20:1215-1228.

45 Treutlein B, Lee QY, Camp JG, Mall M, Koh W, Shariati SA, Sim S, Neff NF, Skotheim JM, Wernig M, Quake SR: Dissecting direct reprogramming from fibroblast to neuron using single-cell RNA-seq. Nature 2016;534: 391-395.

46 Qiu WL, Zhang YW, Feng Y, Li LC, Yang L, Xu CR: Deciphering pancreatic islet $\beta$ cell and a cell maturation pathways and characteristic features at the single-cell level. Cell Metab 2017;25:1194-1205.

47 Bach K, Pensa S, Grzelak M, Hadfield J, Adams DJ, Marioni JC, Khaled WT: Differentiation dynamics of mammary epithelial cells revealed by single-cell RNA sequencing. Nat Commun 2017;8:2128.

48 Athanasiadis EI, Botthof JG, Andres H, Ferreira L, Lio P, Cvejic A: Single-cell RNA-sequencing uncovers transcriptional states and fate decisions in haematopoiesis. Nat Commun 2017;8:2045.

49 Raj B, Wagner DE, McKenna A, Pandey S, Klein AM, Shendure J, Gagnon JA, Schier AF: Simultaneous single-cell profiling of lineages and cell types in the vertebrate brain. Nat Biotechnol 2018;36:442-450.

50 Patel AP, Tirosh I, Trombetta JJ, Shalek AK, Gillespie SM, Wakimoto H, Cahill DP, Nahed BV, Curry WT, Martuza RL, Louis DN, Rozenblatt-Rosen $\mathrm{O}$, Suvà $\mathrm{ML}$, Regev $\mathrm{A}$, Bernstein BE: Single-cell RNA-seq highlights intratumoral heterogeneity in primary glioblastoma. Science 2014;344:1396-1401.

51 Tirosh I, Izar B, Prakadan SM, Wadsworth MH, Treacy D, Trombetta JJ, Rotem A, Rodman C, Lian C, Murphy G, Fallahi-Sichani M, Dutton-Regester K, Lin JR, Cohen O, Shah P, Lu D, Genshaft AS, Hughes TK, Ziegler CG,
Kazer SW, Gaillard A, Kolb KE, Villani AC, Johannessen CM, Andreev AY, van Allen EM, Bertagnolli M, Sorger PK, Sullivan RJ, Flaherty KT, Frederick DT, Jané-Valbuena J, Yoon $\mathrm{CH}$, Rozenblatt-Rosen O, Shalek AK, Regev A, Garraway LA: Dissecting the multicellular ecosystem of metastatic melanoma by single-cell RNA-seq. Science 2016;352:189196.

52 Giustacchini A, Thongjuea S, Barkas N, Woll PS, Povinelli BJ, Booth CAG, Sopp P, Norfo R, Rodriguez-Meira A, Ashley N, Jamieson L, Vyas P, Anderson K, Segerstolpe Å, Qian H, Olsson-Strömberg U, Mustjoki S, Sandberg R, Jacobsen SEW, Mead AJ: Single-cell transcriptomics uncovers distinct molecular signatures of stem cells in chronic myeloid leukemia. Nat Med 2017;23:692-702.

53 Miyamoto DT, Zheng Y, Wittner BS, Lee RJ, Zhu H, Broderick KT, Desai R, Fox DB, Brannigan BW, Trautwein J, Arora KS, Desai N, Dahl DM, Sequist LV, Smith MR, Kapur R, Wu CL, Shioda T, Ramaswamy S, Ting DT, Toner M, Maheswaran S, Haber DA: RNASeq of single prostate CTCs implicates noncanonical Wnt signaling in antiandrogen resistance. Science 2015;349:1351-1356.

54 Gaublomme JT, Yosef N, Lee Y, Gertner RS, Yang LV, Wu C, Pandolfi PP, Mak T, Satija R, Shalek AK, Kuchroo VK, Park H, Regev A: Single-cell genomics unveils critical regulators of Th17 cell pathogenicity. Cell 2015;163: 1400-1412.

55 Van der Wijst MGP, Brugge $\mathrm{H}$, de Vries DH, Deelen P, Swertz MA; LifeLines Cohort Study; BIOS Consortium, Franke L: Singlecell RNA sequencing identifies celltype-specific cis-eQTLs and co-expression QTLs. Nat Genet 2018;50:493-497.

56 Hou Y, Guo H, Cao C, Li X, Hu B, Zhu P, Wu X, Wen L, Tang F, Huang Y, Peng J: Singlecell triple omics sequencing reveals genetic, epigenetic, and transcriptomic heterogeneity in hepatocellular carcinomas. Cell Res 2016; 26:304-319.

57 Hu Y, Huang K, An Q, Du G, Hu G, Xue J, Zhu X, Wang CY, Xue Z, Fan G: Simultaneous profiling of transcriptome and DNA methylome from a single cell. Genome Biol 2016;17:88

58 Clark SJ, Argelaguet R, Kapourani CA, Stubbs TM, Lee HJ, Alda-Catalinas C, Krueger F, Sanguinetti G, Kelsey G, Marioni JC, Stegle O, Reik W: scNMT-seq enables joint profiling of chromatin accessibility DNA methylation and transcription in single cells. Nat Commun 2018;9:781.

59 Stoeckius M, Hafemeister C, Stephenson W, Houck-Loomis B, Chattopadhyay PK, Swerdlow H, Satija R, Smibert P: Simultaneous epitope and transcriptome measurement in single cells. Nat Methods 2017;14:865-868.

60 Peterson VM, Zhang KX, Kumar N, Wong J, Li L, Wilson DC, Moore R, McClanahan TK, Sadekova S, Klappenbach JA: Multiplexed quantification of proteins and transcripts in single cells. Nat Biotechnol 2017;35:936-939.
61 Fan X, Zhang X, Wu X, Guo H, Hu Y, Tang F, Huang Y: Single-cell RNA-seq transcriptome analysis of linear and circular RNAs in mouse preimplantation embryos. Genome Biol 2015; 16:148.

62 Hayashi T, Ozaki H, Sasagawa Y, Umeda M, Danno H, Nikaido I: Single-cell full-length total RNA sequencing uncovers dynamics of recursive splicing and enhancer RNAs. Nat Commun 2018;9:619.

63 Faridani OR, Abdullayev I, Hagemann-Jensen M, Schell JP, Lanner F, Sandberg R: Single-cell sequencing of the small-RNA transcriptome. Nat Biotechnol 2016;34:12641266.

64 Habib N, Li Y, Heidenreich M, Swiech L, Avraham-Davidi I, Trombetta JJ, Hession C, Zhang F, Regev A: Div-Seq: single-nucleus RNA-Seq reveals dynamics of rare adult newborn neurons. Science 2016;353:925-928.

65 Dixit A, Parnas O, Li B, Chen J, Fulco CP, Jerby-Arnon L, Marjanovic ND, Dionne D, Burks T, Raychowdhury R, Adamson B, Norman TM, Lander ES, Weissman JS, Friedman $\mathrm{N}$, Regev A: Perturb-seq: dissecting molecular circuits with scalable single-cell RNA profiling of pooled genetic screens. Cell 2016;167: 1853-1866.e17.

66 Adamson B, Norman TM, Jost M, Cho MY, Nuñez JK, Chen Y, Villalta JE, Gilbert LA, Horlbeck MA, Hein MY, Pak RA, Gray AN, Gross CA, Dixit A, Parnas O, Regev A, Weissman JS: A multiplexed single-cell CRISPR screening platform enables systematic dissection of the unfolded protein response. Cell 2016;167:1867-1882.e21.

67 Jaitin DA, Weiner A, Yofe I, Lara-Astiaso D, Keren-Shaul H, David E, Salame TM, Tanay A, van Oudenaarden A, Amit I: Dissecting immune circuits by linking CRISPR-pooled screens with single-cell RNA-seq. Cell 2016; 167:1883-1896.e15.

68 Datlinger P, Rendeiro AF, Schmidl C, Krausgruber T, Traxler P, Klughammer J, Schuster LC, Kuchler A, Alpar D, Bock C: Pooled CRISPR screening with single-cell transcriptome readout. Nat Methods 2017;14:297-301.

69 Schröppel B, Huber S, Horster M, Schlöndorff D, Kretzler M: Analysis of mouse glomerular podocyte mRNA by single-cell reverse transcription-polymerase chain reaction. Kidney Int 1998;53:119-124.

70 Huber S, Schröppel B, Kretzler M, Schlöndorff D, Horster M: Single cell RT-PCR analysis of ClC-2 mRNA expression in ureteric bud tip. Am J Physiol 1998;274:F951-F957.

71 Nissant A, Lourdel S, Baillet S, Paulais M, Marvao P, Teulon J, Imbert-Teboul M: Heterogeneous distribution of chloride channels along the distal convoluted tubule probed by single-cell RT-PCR and patch clamp. Am J Physiol Renal Physiol 2004;287:F1233F1243.

$72 \mathrm{Lu}$ Y, Ye Y, Yang Q, Shi S: Single-cell RNAsequence analysis of mouse glomerular mesangial cells uncovers mesangial cell essential genes. Kidney Int 2017;92:504-513. 
$73 \mathrm{Lu}$ Y, Ye Y, Bao W, Yang Q, Wang J, Liu Z, Shi S: Genome-wide identification of genes essential for podocyte cytoskeletons based on single-cell RNA sequencing. Kidney Int 2017; 92:1119-1129.

74 Der E, Ranabothu S, Suryawanshi H, Akat KM, Clancy R, Morozov P, Kustagi M, Czuppa M, Izmirly P, Belmont HM, Wang T, Jordan N, Bornkamp N, Nwaukoni J, Martinez J, Goilav B, Buyon JP, Tuschl T, Putterman C: Single cell RNA sequencing to dissect the molecular heterogeneity in lupus nephritis. JCI Insight 2017;2:93009.

75 Tang Q, Iyer S, Lobbardi R, Moore JC, Chen H, Lareau C, Hebert C, Shaw ML, Neftel C, Suva ML, Ceol CJ, Bernards A, Aryee M, Pinello L, Drummond IA, Langenau DM: Dissecting hematopoietic and renal cell heterogeneity in adult zebrafish at single-cell resolution using RNA sequencing. J Exp Med 2017; 214:2875-2887.
76 Chen L, Lee JW, Chou CL, Nair AV, Battistone MA, Păunescu TG, Merkulova M, Breton S, Verlander JW, Wall SM, Brown D, Burg MB, Knepper MA: Transcriptomes of major renal collecting duct cell types in mouse identified by single-cell RNA-seq. Proc Natl Acad Sci USA 2017;114:E9989-E9998.

77 Adam M, Potter AS, Potter SS: Psychrophilic proteases dramatically reduce single-cell RNA-seq artifacts: a molecular atlas of kidney development. Development 2017;144:36253632.

78 Magella B, Adam M, Potter AS, Venkatasubramanian M, Chetal K, Hay SB, Salomonis N, Potter SS: Cross-platform single cell analysis of kidney development shows stromal cells express Gdnf. Dev Biol 2018;434:36-47.

79 Park J, Shrestha R, Qiu C, Kondo A, Huang S, Werth M, Li M, Barasch J, Suszták K: Singlecell transcriptomics of the mouse kidney reveals potential cellular targets of kidney disease. Science 2018;360:758-763.
80 Karaiskos N, Rahmatollahi M, Boltengagen A, Liu H, Hoehne M, Rinschen M, Schermer B, Benzing T, Rajewsky N, Kocks C, Kann M, Müller RU: A single-cell transcriptome atlas of the mouse glomerulus. J Am Soc Nephrol 2018;29:2060-2068.

81 Lin F, Moran A, Igarashi P: Intrarenal cells, not bone marrow-derived cells, are the major source for regeneration in postischemic kidney. J Clin Invest 2005;115:1756-1764.

82 Iwano M, Plieth D, Danoff TM, Xue C, Okada H, Neilson EG: Evidence that fibroblasts derive from epithelium during tissue fibrosis. J Clin Invest 2002;110:341-350.

83 Appel D, Kershaw DB, Smeets B, Yuan G, Fuss A, Frye B, Elger M, Kriz W, Floege J, Moeller MJ: Recruitment of podocytes from glomerular parietal epithelial cells. J Am Soc Nephrol 2009;20:333-343.

84 Romagnani P, Rinkevich Y, Dekel B: The use of lineage tracing to study kidney injury and regeneration. Nat Rev Nephrol 2015;11:420-431. 